OPEN ACCESS

Edited by:

Leonard Peruski,

US Centers for Disease Control and

Prevention, USA

Reviewed by:

Peter Bergman,

Karolinska Institutet, Sweden Marquita Vernescia Gittens-St. Hilaire

University of the West Indies,

Barbados

*Correspondence:

Mingxi Wang

mxwang@hqu.edu.cn

Desong Ming

mds6430@126.com

Shicheng Chen

shicheng@msu.edu

${ }^{\dagger}$ These authors have contributed equally to this work

Specialty section:

This article was submitted to

Infectious Diseases,

a section of the journal

Frontiers in Microbiology

Received: 20 November 2016

Accepted: 23 February 2017

Published: 08 March 2017

Citation:

Hu S, Jiang T, Zhang $X$, Zhou Y, Yi Z

Wang $Y$, Zhao S, Wang M, Ming D

and Chen S (2017) Elizabethkingia anophelis Isolated from Patients with Multiple Organ Dysfunction Syndrome and Lower Respiratory Tract Infection:

Report of Two Cases and Literature

Review. Front. Microbiol. 8:382. doi: 10.3389/fmicb.2017.00382

\section{Elizabethkingia anophelis Isolated from Patients with Multiple Organ Dysfunction Syndrome and Lower Respiratory Tract Infection: Report of Two Cases and Literature Review}

\author{
Shaohua $\mathrm{Hu}^{1+}$, Tao Jiang ${ }^{1+}$, Xia Zhang ${ }^{2}$, Yajun Zhou ${ }^{1}$, Zhengjun $\mathrm{Yi}^{2}$, Youxi Wang ${ }^{3}$, \\ Sishou Zhao ${ }^{3}$, Mingxi Wang ${ }^{1,2 *}$, Desong Ming ${ }^{4 *}$ and Shicheng Chen ${ }^{5 *}$ \\ ${ }^{1}$ Yun Leung Laboratory for Molecular Diagnostics, School of Biomedical Sciences, Huaqiao University, Xiamen, China, \\ ${ }^{2}$ Department of Medical Laboratory, Institute of Nanomedicine Technology, Weifang Medical University, Weifang, China, \\ ${ }^{3}$ Department of Information, Quanzhou First Hospital Affiliated to Fujian Medical University, Fujian, China, ${ }^{4}$ Department of \\ Clinical Laboratory, Quanzhou First Hospital Affiliated to Fujian Medical University, Fujian, China, ${ }^{5}$ Department of Microbiology \\ and Molecular Genetics, Michigan State University, East Lansing, MI, USA
}

Elizabethkingia anophelis, originally discovered from Anopheles mosquito gut, is an emerging pathogen, especially in immunocompromised patients. We isolated two strains of $E$. anophelis from two separate patients with multiple organ dysfunction syndrome and lower respiratory tract infection. In this paper, we reviewed the status of $E$. anophelis infection and its antibiotics resistance from reported cases.

Keywords: E. anophelis, emerging pathogen, multiple organ dysfunction syndrome, lower respiratory tract infection, antibiotics resistance

\section{BACKGROUND}

Elizabethkingia, a Gram-negative, aerobic, rod-shaped bacterium, belongs to the family Flavobacteriaceae within the phylum Bacteroidetes. Four species are assigned to this genus, including Elizabethkingia meningoseptica, E. anophelis, Elizabethkingia miricola, and Elizabethkingia endophytica, with the first three being considered to be medically important (Kukutla et al., 2013, 2014; Lau et al., 2015; Breurec et al., 2016). E. anophelis was initially isolated from the midgut of mosquito Anopheles gambiae in 2011 (Kämpfer et al., 2011). Later, it was found in the environment (water and soil) and health care settings (Balm et al., 2013; Teo et al., 2013). It rarely causes infections in healthy people while it is pathogenic to immunocompromised patients with a high mortality rate (Teo et al., 2013; Lau et al., 2016; https://www.cdc.gov/elizabethkingia/ outbreaks/). The first reported case of E. anophelis infection was meningitis in an 8-day-old girl delivered by cesarean section in the Central African Republic in 2011 (Frank et al., 2013). It also caused nosocomial outbreak in two intensive-care units from a hospital in Singapore in 2012 (Teo et al., 2013). By conventional phenotypic test, an epidemiological survey of bacteremia from five regional hospitals in Hong Kong (from 2004 to 2013) identified 17 cases of E. anophelis bacteremia (Lau et al., 2016). E. anophelis was implicated in recent outbreaks of infections in the Midwest regions. In Wisconsin, 63 confirmed and 4 possible infections were reported between November 1, 2015 and January 11, 2017. Among these cases, a total of 19 deaths (estimated mortality rate, 28\%) were found (https://www.dhs.wisconsin.gov/disease/elizabethkingia.htm). At least $10 \mathrm{E}$. anophelis infections were reported in Illinois (between January 2014 and April 2016) and one in Michigan in March 2016 (https://www.cdc.gov/elizabethkingia/outbreaks/). Most patients 
were over 65 years old with serious underlying diseases (https:// www.cdc.gov/elizabethkingia/outbreaks/). We report here two cases of E. anophelis infection at Quanzhou First Hospital, Fujian, China.

\section{CASE PRESENTATION}

\section{Case 1}

An 82-year-old male patient presenting with lethargy in consciousness, malnutrition, multiple bedsores [an $8 \times 10$ $\mathrm{cm}^{2}$ at level I (containing a $5 \times 6 \mathrm{~cm}^{2}$ at level II) on the sacrococcygeal region, two $5 \times 5 \mathrm{~cm}^{2}$ at level $\mathrm{I}$ on the both heel, two $2 \times 2 \mathrm{~cm}^{2}$ at level I on both external malleolus] was admitted on December 14, 2009. He had a history of surgery for esophagus cancer at 73 years old, hypertension at level III, two hospitalizations due to lower respiratory tract infections (treated by azithromycin, cefoperazone/tazobactam, levofloxacin, meropenem, linezolid, piperacillin/tazobactam, and ciprofloxacin) 4 months before this final hospitalization. He demonstrated expectoration and shortness of breath for 7 days before admission. The blood gas analysis and blood biochemical test at admission showed respiratory acidosis $\left(\mathrm{pCO}_{2}\right.$

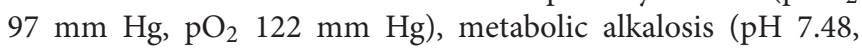
$\mathrm{HCO}_{3}^{-}>60 \mathrm{mmol} / \mathrm{L}, \mathrm{CO}_{2} 36.8 \mathrm{mmol} / \mathrm{L}$ ), very low blood $\mathrm{K}^{+}(2.1 \mathrm{mmol} / \mathrm{L})$, renal dysfunction (BUN $\left.23.9 \mathrm{mmol} / \mathrm{L}\right)$, and extremely low $\mathrm{Cl}^{-}(68.6 \mathrm{mmol} / \mathrm{L})$. He came into deep coma with BP 58/34 mmHg within $2 \mathrm{~h}$ after admission. Emergency salvage was given, including assisted ventilation, raising BP by dopamine and L-noradrenaline, supplement of potassium chloride, and anti-infection treatment with piperacillin/tazobactam and ciprofloxacin. In the subsequent 2 days, he recovered from coma gradually, but presented clear sign of lung infection (fever, moist rale sound in both sides of lower lung lobes, blood WBC $9.8 \times 10^{9} / \mathrm{L}$, NEUT 94.61\%, CRP $146 \mathrm{mg} / \mathrm{L}$ ). Diagnoses of shock, multiple organ dysfunction syndrome (MODS), and lower respiratory tract infection were made. The infection and the respiratory acidosis combined with metabolic alkalosis lasted for nearly the whole 52 day-hospitalization. On the 45th day of hospitalization, a fistula between the esophagus and trachea was found through gastroscopy and further verified by bronchoscopy. It was treated with tracheal stenting 2 days later. However, $5 \mathrm{~h}$ after posttracheal stenting, sudden cardiac arrest occurred, complicated with a loss of spontaneous breathing, then he went into coma (combined with dilated pupils and disappeared light reflex of both sides) again. $\mathrm{BP}$ and $\mathrm{SpO}_{2}$ could not be measured while his pulse and spontaneous breathing returned $5 \mathrm{~min}$ after salvage. $\mathrm{BP}, \mathrm{SpO}_{2}$, and light reflex of both pupils returned to normal gradually within the subsequent $5 \mathrm{~h}$. However, the patient was still in coma and was discharged after 7 days at his family's request to terminate treatment.

During the hospitalization, the separately or jointed isolated pathogenic microbes for the lung infection included Acinetobacter calcoaceticus-Acinetobacter baumannii complex (7 times isolated from sputum, 7 TIFS), Acinetobacter baumannii (4 TIFS), Enterobacter cloacae (3 TIFS), Pseudomonas aeruginosa (2 TIFS), Serratia marcescens (2 TIFS), molds
(2 TIFS), and E. anophelis (7 TIFS) (named as 12012 PRCM). E. anopheles 12012 PRCM was verified by $16 \mathrm{~S}$ rRNA gene sequencing (GenBank accession no. KR349263) of the genomic DNA with the forward primer $27 \mathrm{~F}\left(5^{\prime}-\right.$ AGAGTTTGATCCTGGCTCAG-3') and the reverse primer 1492R (5'-GGTTACCTTGTTACGACTT-3'; Lane, 1991). It was resistant to all the 20 antibiotics in the antibiotic susceptibility tests (AST) panel, including $\beta$-lactams (ampicillin, piperacillin, ampicillin-sulbactam, piperacillin-tazobactam, ceftazidime, cefotaxime, cephazolin, cefepime, aztreonam, imipenem, and meropenem), aminoglycosides (amikacin, gentamicin), tetracycline, chloramphenicol, and polymyxin, quinolones (ciprofloxacin, levofloxacin, moxifloxacin), and sulfamethoxazole-trimethoprim. The strain was also resistant to another antibiotic amoxicillin-clavulanate tested. To investigate the resistance mechanisms, extended spectrum $\beta$-lactamase (ESBL) detection was performed on the E. anophelis 12012 PRCM using BD's PhoenixTM-100 Automated Microbiology System with the NMIC/ID-4 panel (Becton, Dickinson, and Company; Stefaniuk et al., 2003; Liu et al., 2005). The cephalosporin test was positive and the carbapenem-hydrolyzing $\beta$-lactamases and carbapenemases (CHBLs) were detected. In addition, we found this strain was co-positive between EDTA (Ethylenediaminetetraacetic acid) and imipenem. These laboratory findings indicated strain 12012 PRCM could produce metal-beta-lactamase, possibly conferring the $\beta$-lactamases resistance.

\section{Case 2}

A 47-year-old male with hypotension (BP 95/55 mmHg) was admitted to our hospital on August 21, 2015. He had history of exacerbated abdominal pain and abdominal distension for more than 10 days, shortness of breath for 3 days, complicated with stagnated exhaust and defecation. Before admission, his pressure was maintained by continuous pumping of L-noradrenaline and dopamine. Physical examination found weak breath sounds in both sides of lung lobes and severe subcutaneous edema on whole body. The arterial blood gas analysis displayed acidosis $\left(\mathrm{pH}\right.$ 7.20, $\mathrm{pCO}_{2} 11.1 \mathrm{mmHg}, \mathrm{pO}_{2} 99 \mathrm{mmHg}, \mathrm{HCO}_{3}^{-}-8.6$ $\mathrm{mmol} / \mathrm{L}, \mathrm{BE}-23.3 \mathrm{mmol} / \mathrm{L})$. He had a low white blood cell count $(\mathrm{WBC})\left(2.35 \times 10^{9} / \mathrm{L}\right)$. Blood biochemical test showed several abnormal results (glucose $8.86 \mathrm{mmol} / \mathrm{L}$, BUN 18.93 $\mathrm{mmol} / \mathrm{L}$, creatinine $271 \mu \mathrm{mol} / \mathrm{L}$, GFR $24.20 \mathrm{ml} / \mathrm{min}$, AST 827 U/L, ALT 197 U/L, albumin $30.5 \mathrm{~g} / \mathrm{L}$, T-BIL $29.5 \mu \mathrm{mol} / \mathrm{L}, \mathrm{D}-\mathrm{BIL}$ $12.8 \mu \mathrm{mol} / \mathrm{L}$, total osmotic pressure $280 \mathrm{mOsm} / \mathrm{L}$ ). Ultrasound imaging demonstrated liver cirrhosis, echo enhancement in parenchyma of both kidneys and effusion around left renal. Computed tomography scanning revealed inflammatory changes within both sides of the lower lung lobes, severe effusion within bilateral pleural, abdominal, and pelvic cavity, edema within subcutaneous soft tissue of chest and abdominal wall. Diagnoses of shock, MODS, electrolyte imbalances (low sodium, low calcium), diabetes and pulmonary infection were made. Within $16 \mathrm{~h}$ after admission, the patient further presented oliguria and aggravated during the 12-day hospitalization. On the 3rd day, hepatic virus infection [with positive HBsAg, anti-HBe antibody, and anti-HBc antibody (IgG)], abnormal 
blood coagulation [prothrombin time (PT) $16.7 \mathrm{~s}$, partial prothrombin time (APTT) $67.8 \mathrm{~s}$, fibrinogen (FIB) $1.75 \mathrm{~g} / \mathrm{L}, \mathrm{D}-$ dimer $8.32 \mathrm{mg} / \mathrm{L}$, international normalized ratio (INR) 1.43], and thrombocytopenia $\left(84 \times 10^{9} / \mathrm{L}\right)$ were diagnosed, with the latter lasting for the remaining hospitalization. The treatment strategies included drainage of bilateral pleural effusion, maintaining blood pressure as previously, improving liver function, daily hemodialysis, management of electrolyte, insulin injection and improving nutrition. The empirical antimicrobial therapy was started with IV piperacillin and tazobactam during the first 3 days of hospitalization, then changed to IV imipenem and cilastatin sodium for 1 day and further combined with vancomycin; the latter lasted for the remaining 8 days of hospitalization. The patient died of sudden cardiac arrest.

By using BD's Phoenix ${ }^{\mathrm{TM}}-100$ Automated Microbiology System with the NMIC/ID-4 panel as Case 1, we repeatedly isolate E. meningoseptica (on the 7th and 9th day of hospitalization respectively) and Stenotrophomonas maltophilia (on the 8th and 9th day of hospitalization respectively) from sputum cultures. Later, E. meningoseptica was verified as E. anophels (named as N107618, GenBank accession No. KX775952) by $16 \mathrm{~S}$ rRNA gene sequencing as Case 1. E. anophelis N107618 was resistant to 16 out of 20 antibiotics in the same AST panel used to test the E. anophelis 12012 PRCM, and was only susceptible to ciprofloxacin, levofloxacin, moxifloxacin, and sulfamethoxazole-trimethoprim.

\section{DISCUSSION}

E. anophelis was an opportunistic pathogen but it could result in a high mortality rate (Teo et al., 2013; Lau et al., 2016; https://www. cdc.gov/elizabethkingia/outbreaks/). The number of E. anophelis infections was possibly underestimated because E. anophelis were often misidentified as E. meningoseptica by conventional identification methods (Kämpfer et al., 2011; Teo et al., 2013, 2014; Nicholson et al., 2016). Identification of E. anophelis by the matrix assisted laser desorption-ionization time-of-flight (MALDI-TOF) mass spectrometry will be helpful if E. anophelis reference is added in the database (Teo et al., 2013; Lau et al., 2016). 16S rRNA gene sequencing should be performed as a routine bacterial identification tool in the medical laboratories without MALDI-TOF mass spectrometry (Teo et al., 2013; Lau et al., 2016).

The high mortality rate of $E$. anophelis infection was partly due to its property of multidrug-resistance. E. anophelis N107618 was resistant to 16 out of 20 antibiotics while strain 12012 PRCM was resistant to all the 20 antibiotics in the AST panel, indicating that our two clinical E. anophelis isolates were multidrugresistant. Furthermore, our tests showed that the isolate E. anophelis 12012 PRCM produced metallo-beta-lactamase(s). $\beta$-lactams resistance has been shown in many clinical important Elizabethkingia isolates (Frank et al., 2013; Lau et al., 2015, 2016). Notably, all clinical E. anophelis isolates, except for our strain 12012 PRCM, were susceptible to one or more quinolones such as ciprofloxacin or moxifloxacin (Frank et al., 2013; Lau et al., 2015). Therefore, ciprofloxacin or moxifloxacin might be considered as empirical antibiotic therapy of E. anophelis infections. For example, Frank et al. reported that a clinical isolate was resistant to all $\beta$-lactams tested (amoxicillin, cefotaxime, ceftazidime, cefepime, and imipenem) except for piperacillin and aminoglycosides (amikacin, tobramycin, and gentamicin) while it was susceptible to moxifloxacin (Frank et al., 2013). Similarly, three clinical $E$. anophelis strains were resistant to $\beta$-lactams aminoglycosides, tetracycline, and chloramphenicol while they were susceptible to pipercillin, cefoperazone-sulbactam, trimethoprim-sulfamethoxazole, ciprofloxacin, moxifloxacin, and rifampin (Lau et al., 2015). Moreover, the survey of $17 \mathrm{E}$. anophelis bacteremia demonstrated that they were resistant or intermediate-resistant to imipenem, amikacin, gentamicin, and tobramycin, but they were susceptible to ceftazidime, piperacillin, and rifampicin (Lau et al., 2016).

Next generation sequencing (NGS) techniques greatly contribute to our understanding of drug resistance and infectivity of E. anophelis. For example, 16 and 19 antibiotic resistance genes were found from the core and accessory genomes of E. anophelis, respectively (Teo et al., 2014). The genome of E. anophelis NUHP1 contained 14 antibiotic resistant genes that correlated to its multi-drug resistance (Li et al., 2015). Breurec et al. reported that at least 17 antimicrobial resistance genes in E. anophelis consisted of metallo-beta-lactamase genes $b l a_{\mathrm{blaB}}$ and $b l a_{\mathrm{GOB}}$ (conferring $\beta$-lactams resistance), subclass $\mathrm{B} 1$ beta-lactamase gene, efflux systems, conserved chloramphenicol acetyltransferase (CAT and AAC3-I) gene, a tet $X$ gene (encoding for a tetracycline inactivating enzyme) and $\operatorname{Lin} F$ gene (encoding lincosamide resistance protein; Breurec et al., 2016). Genome sequencing analysis showed that $E$. anophelis processed virulence genes encoding cell wall hydrolase A, listeriolysin O (LLO), phospholipase (PlcA), virulence cluster protein $\mathrm{B}(\mathrm{VclB})$, and arylsulfatase (Lau et al., 2015; Breurec et al., 2016). These virulence factors possibly contribute to the invading of the host, escape from phagocytosis, surviving in the secondary vacuole of macrophages, and propagating in bloodstreams (Lau et al., 2015; Breurec et al., 2016).

\section{CONCLUSION}

E. anophelis is an emerging opportunistic pathogen. It often leads to a high mortality rate because of its multiple antibiotic resistance. Therefore, E. anophelis infections should be considered clinically significant. Investigation of antibiotic resistance mechanisms are warranted.

\section{ETHICS STATEMENT}

The written informed consents were obtained from the patients or their relatives/guardians. This work was already approved by the Ethics Committee of Quanzhou First Hospital.

\section{AUTHOR CONTRIBUTIONS}

SH and TJ contributed equally to this work. All of authors wrote the papers. SH conducted the test. MW, DM, and SC guided the testing and experiments. 


\section{ACKNOWLEDGMENTS}

This work was supported by Huaqiao University Graduate Student Scientific Research Innovation Ability Cultivation Plan Projects, the Fong Shu Fook Tong and Fong Yun Wah Foundations (14X30127), the Xiamen Southern Oceanographic

\section{REFERENCES}

Balm, M. N., Salmon, S., Jureen, R., Teo, C., Mahdi, R., Seetoh, T., et al. (2013). Bad design, bad practices, bad bugs: frustrations in controlling an outbreak of Elizabethkingia meningoseptica in intensive care units. J. Hosp. Infect. 85, 134-140. doi: 10.1016/j.jhin.2013.05.012

Breurec, S., Criscuolo, A., Diancourt, L., Rendueles, O., Vandenbogaert, M., Passet, V., et al. (2016). Genomic epidemiology and global diversity of the emerging bacterial pathogen Elizabethkingia anophelis. Sci. Rep. 6:30379. doi: 10.1038/srep30379

Frank, T., Gody, J. C., Nguyen, L. B., Berthet, N., Le Fleche-Mateos, A., Bata, P., et al. (2013). First case of Elizabethkingia anophelis meningitis in the Central African Republic. Lancet 381:1876. doi: 10.1016/S0140-6736(13)60318-9

Kämpfer, P., Matthews, H., Glaeser, S. P., Martin, K., Lodders, N., and Faye, I. (2011). Elizabethkingia anophelis sp. nov., isolated from the midgut of the mosquito Anopheles gambiae. Int. J. Syst. Evol. Microbiol. 61(Pt 11), 2670-2675. doi: 10.1099/ijs.0.026393-0

Kukutla, P., Lindberg, B. G., Pei, D., Rayl, M., Yu, W., Steritz, M., et al. (2013). Draft genome sequences of Elizabethkingia anophelis strains R26T and Ag1 from the midgut of the malaria mosquito Anopheles gambiae. Genome Announc. 1, e01030-e01013. doi: 10.1128/genomeA.01030-13

Kukutla, P., Lindberg, B. G., Pei, D., Rayl, M., Yu, W., Steritz, M., et al. (2014). Insights from the genome annotation of Elizabethkingia anophelis from the malaria vector Anopheles gambiae. PLoS ONE 9:e97715. doi: 10.1371/journal.pone.0097715

Lane, D. J. (1991). “16S/23S rRNA sequencing," in Nucleic Acid Techniques in Bacterial Systematics, eds E. Stackebrandt and M. Goodfellow (Chichester: John Wiley \& Sons), 115-175.

Lau, S. K., Chow, W. N., Foo, C. H., Curreem, S. O., Lo, G. C., Teng, J. L., et al. (2016). Elizabethkingia anophelis bacteremia is associated with clinically significant infections and high mortality. Sci. Rep. 6:26045. doi: $10.1038 /$ srep 26045

Lau, S. K., Wu, A. K., Teng, J. L., Tse, H., Curreem, S. O., Tsui, S. K., et al. (2015). Evidence for Elizabethkingia anophelis transmission from mother to infant, Hong Kong. Emerg. Infect. Dis. 21, 232-241. doi: 10.3201/eid2102.140623
Center (14GYY008NF08), the Natural Science Fund Project of Fujian Province (2015J01514), the Technology Planning Projects of Quanzhou Social Development Fields (2014Z24), and the Major Support Research Project of National Key Colleges Construction of Quanzhou Medical College (2013A13).

Li, Y., Liu, Y., Chew, S. C., Tay, M., Salido, M. M., Teo, J., et al. (2015). Complete genome sequence and transcriptomic analysis of the novel pathogen Elizabethkingia anophelis in response to oxidative stress. Genome Biol. Evol. 7, 1676-1685. doi: 10.1093/gbe/evv101

Liu, Z. K., Ling, T. K., and Cheng, A. F. (2005). Evaluation of the BD phoenix automated microbiology system for identification and antimicrobial susceptibility testing of common clinical isolates. Med. Princ. Pract. 14, 250-254. doi: 10.1159/000085744

Nicholson, A. C., Whitney, A. M., Emery, B. D., Bell, M. E., Gartin, J. T., Humrighouse, B. W., et al. (2016). Complete genome sequences of four strains from the 2015-2016 Elizabethkingia anophelis outbreak. Genome Announc. 4, e00563-00564. doi: 10.1128/genomeA.00563-16

Stefaniuk, E., Baraniak, A., Gniadkowski, M., and Hryniewicz, W. (2003). Evaluation of the BD Phoenix automated identification and susceptibility testing system in clinical microbiology laboratory practice. Eur. J. Clin. Microbiol. Infect. Dis. 22, 479-485. doi: 10.1007/s10096-003-0962-y

Teo, J., Tan, S. Y., Liu, Y., Tay, M., Ding, Y., Li, Y., et al. (2014). Comparative genomic analysis of malaria mosquito vector-associated novel pathogen Elizabethkingia anophelis. Genome Biol. Evol. 6, 1158-1165. doi: 10.1093/gbe/evu094

Teo, J., Tan, S. Y., Tay, M., Ding, W., Kjelleberg, S., Givskov, M., et al. (2013). First case of $E$. anophelis outbreak in an intensive-care unit. Lancet 382, 855-856. doi: 10.1016/S0140-6736(13)61858-9

Conflict of Interest Statement: The authors declare that the research was conducted in the absence of any commercial or financial relationships that could be construed as a potential conflict of interest.

Copyright (c) 2017 Hu, Jiang, Zhang, Zhou, Yi, Wang, Zhao, Wang, Ming and Chen. This is an open-access article distributed under the terms of the Creative Commons Attribution License (CC BY). The use, distribution or reproduction in other forums is permitted, provided the original author(s) or licensor are credited and that the original publication in this journal is cited, in accordance with accepted academic practice. No use, distribution or reproduction is permitted which does not comply with these terms. 